\title{
Artificial neural network and partial least square in predicting blood hemoglobin using near-infrared spectrum
}

\author{
Mohd Nazrul Effendy Mohd Idrus ${ }^{1}$, Kim Seng Chia ${ }^{2}$ \\ ${ }^{1,2}$ Faculty of Electrical and Electronic Engineering, Universiti Tun Hussein Onn Malaysia, Malaysia \\ ${ }^{2}$ Sensor Devices Research Laboratory, MiNT-SRC, Universiti Tun Hussein Onn Malaysia, Malaysia
}

\begin{tabular}{l} 
Article Info \\
\hline Article history: \\
Received Jan 26, 2019 \\
Revised May 9, 2019 \\
Accepted May 22, 2019 \\
\hline
\end{tabular}

Keywords:

Artificial neural network Hybrid model

Near infrared spectroscopy

Partial least square

\begin{abstract}
Predictive models are crucial in near-infrared (NIR) spectroscopic analysis. Partial least square - artificial neural network (PLS-ANN) is a hybrid method that may improve the performance of prediction in NIR spectroscopic analysis. This study investigates the advantage of PLS-ANN over the wellknown linear and non-linear modelling approaches in spectroscopy analysis that are partial least square (PLS) and artificial neural network (ANN). The results show that ANN that coupled with first order SG derivatives achieved the best prediction with root mean square error of prediction (RMSEP) of $0.3517 \mathrm{gd} / \mathrm{L}$ and coefficient of determination $\left(\mathrm{R}_{\mathrm{p}}^{2}\right)$ of 0.9849 followed by PLS-ANN with RMSEP of $0.4368 \mathrm{gd} / \mathrm{L}$ and $\mathrm{R}_{\mathrm{p}}^{2}$ of 0.9787 , and PLS with RMSEP of $0.4669 \mathrm{gd} / \mathrm{L}$ and $R_{\mathrm{p}}^{2}$ of 0.9727 . This suggests that the spectrum information may unable to be totally represented by the first few latent variables of PLS and a nonlinear model is crucial to model these nonlinear information in NIR spectroscopic analysis.
\end{abstract}

Copyright () 2019 Institute of Advanced Engineering and Science. All rights reserved.

\section{Corresponding Author:}

Kim Seng Chia,

Faculty of Electrical and Electronic Engineering,

Universiti Tun Hussein Onn Malaysia, Malaysia.

Email: kschia@uthm.edu.my

\section{INTRODUCTION}

Nowadays, near-infrared spectroscopy (NIRS) technology showed increasing number of applications in various fields such as medical, chemical, and food analysis [1-3]. Applications that based on NIRS are developed to overcome several factors in the conventional methods which are time-consuming, destructive, and cost-effective. The intensity of the reflection and transmission of the fundamental molecular vibrations of $\mathrm{C}-\mathrm{H}, \mathrm{O}-\mathrm{H}$, and $\mathrm{N}-\mathrm{H}$ produces absorption bands make near-infrared (NIR) useful for analyzing in the biological system [4]. By using a working range of the electromagnetic spectrum (from about $780 \mathrm{~nm}$ to $2500 \mathrm{~nm}$ ), NIRS produces a spectral data which limit the usage based on several factors such as baseline drift, interference resulting in a poor signal to noise ratio, improper wavelength selection, thermal noise, and calibration issues [5]. However, these problems of nonlinearity in spectral data can be solved by using an appropriate predictive modelling and data preprocessing methods.

Hybridization or combination of two predictive modellings is frequently used in spectroscopic analysis to improve the performance of the modelling. The main purpose of these techniques used is to improve weakness that occurs within single models such as nonlinearity, redundant spectral band, and wavelength selection problems. Evolving of predictive modelling technique was a better solution to improve the accuracy of performance compared than recreating new modelling [6]. Furthermore, hybrid models were used to reduce the risk of failure by using the single model by combining several models to obtain more accurate results [7]. However, the hybrid models are not an easy process to be developed and need a deep knowledge to manipulate the models and avoid the wrong parameter to be selected. 
For instance, artificial neural network (ANN) combined with multiple linear regression (MLR) has been developed to overcome the linear modelling deficiency of the traditional artificial neural networks [7]. The proposed models showed a good result in classification for both synthetic and real-life benchmark data sets and the model consistently outperforms with other predictive models such as multilayer perceptron, linear discriminant analysis, quadratic discriminant analysis, K-nearest neighbour, and support vector machines. However, MLR modelling is not a powerful linear model and can be replaced with another better linear model such as PLS. Genetic algorithm (GA) coupled with PLS showed the capability of retrieving components of interest from spectral data [8]. Furthermore, GA combined with ANN shows a reliable method in classifying an egg's freshness [9]. In addition, ANN hybrid with GA was established to predict travel agency air ticket sales revenue [10]. By using genetic operators such as reproduction, mutation and, selection, GA creates a new generation of the population which is better than the generation before. However, with a large number of spectral data, GA method for selection of variable spectral data could lead to a risk of overfitting. PLS combined with back propagation neural network (BPNN) showed effectiveness in overcoming the problems of redundant and nonlinear in spectral data [11]. Number of latent variables (LVs) generated from PLS was used as the input of BPNN to estimate the abundance of minerals on the lunar surface. Furthermore, PLS combined with BPNN achieved the best performance compared with PLS and GA-PLS. However, optimization of a number of LVs and hidden neurons used in this research is not briefly described.

Next, the quality of different models of PLS, ANN, and PLS-ANN to predict consumer interest rating of ready to drink green tea beverages were investigated [9]. PLS-ANN showed a better quality compared to PLS in coefficient of determination and mean square error value. However, the number of variable in the research which is 8 factors is very small to be compared with a variable in NIRS analysis. Whereas, the working range of NIRS is from $780 \mathrm{~nm}$ to $2500 \mathrm{~nm}$ have 390 to 1250 variables with a $2 \mathrm{~nm}$ interval. A combination of PLS and ANN also was applied in management and chemical analysis showed satisfy result $[11,12]$. Even though the limitation of of PLS has been rectified using the PLS-ANN, there is no comparison among PLS, ANN, and PLS-ANN. Therefore, this study compares three types of predictive modelling i.e. linear model (PLS), nonlinear model (ANN), and a hybrid model (PLS-ANN) in predicting hemoglobin concentration using near-infrared spectral data. We also investigated the optimal number of LVs and hidden neurons to achieve optimal prediction of performance.

\section{MATERIAL AND METHODS}

\subsection{Samples and References}

The origin spectral dataset was adopted from IDRC shootout 2010 provided by Karl Norris. The Blood samples were analyzed with a NIRSystems 6500 spectrometer from 1990 to 1992. All spectral data for calibration and testing have 700 variables, were measured with a range of wavelengths that span the infrared spectrum from 1100 to $2498 \mathrm{~nm}$ wavelength with a $2 \mathrm{~nm}$ interval. The dataset contains 231 sets of calibration and 194 testing data sets to measure predictive accuracy of the modelling. Table 1 shows the summary of descriptive characteristics of the calibration and testing references data. The characteristics of calibration and testing data indicate that extrapolation samples were used for modelling. Therefore, an appropriate modelling technique needs to be implemented to predict the out of range data from the testing process. IDRC shootout 2010 has used the same extrapolation data in the tournament [13].

Table 1. Descriptive statistics of the blood hemoglobin

\begin{tabular}{ccccccc}
\hline Data & $\mathrm{n}$ & Min $(\mathrm{g} / \mathrm{dL})$ & Max $(\mathrm{g} / \mathrm{dL})$ & Mean $(\mathrm{g} / \mathrm{dL})$ & Median $(\mathrm{g} / \mathrm{dL})$ & Std \\
\hline Calibration & 231 & 10.30 & 17.30 & 13.78 & 13.70 & 1.66 \\
Testing & 194 & 6.50 & 18.20 & 12.20 & 12.25 & 2.83 \\
Total & 425 & 6.50 & 18.20 & 13.06 & 13.40 & 2.40 \\
\hline
\end{tabular}

\subsection{Data Preprocessing}

Savitzky-golay (SG) preprocessing method was used as a pretreatment process to remove unwanted signals such as signal to noise ratio, baseline shift effect, and slope affected from spectral data. By using a specific odd number of frame length, a single set of SG coefficient with the same number of frame length can be applied to all subsets' data to evaluate new smoothed or derivatives signal of central point data of each subset. Moreover, an optimal number of frame length should be optimized to achieve an optimum of prediction performance [13, 14]. Raw spectral data of hemoglobin was treated with a different type of SG preprocessing such as smoothing, first order, and second order sg derivatives [15]. SG coefficient can be applied to each subset data to obtain new treated data by using Equation (1). 


$$
Y_{j}=(C \otimes y)_{j}=\sum_{i=-\frac{m-1}{2}}^{\frac{m-1}{2}} C_{i} Y_{i+j}, \frac{m-1}{2} \leq j \leq n-\frac{m-1}{2}
$$

where $m$ and $n$ is measured number frame length and total number of variables. Although, $C_{i}$ is the set of sg coefficient. While, $Y_{i+j}$ and $Y_{j}$ is a related set of data before and observed value after the treatment process.

\subsection{Partial Least Square}

General concept idea behind of PLS modelling is to decompose both the design matrix predictor X and matrix of response Y as Equations (2) and (3)

$$
\begin{aligned}
& X=T P^{T} \\
& Y=U Q^{T}
\end{aligned}
$$

where $X$ is an $n \times m$ matrix of predictors, $Y$ is an $n \times p$ matrix of response.T and $U$ is $n \times l$ matrix that are projections of $X$ score and $Y$ score respectively. $P$ and $Q$ are $m \times l$ and $p \times l$ orthogonal loading matrices respectively. The algorithm will yield the PLS regression estimates $B$ and $B_{o}$ after estimating the factor and loading matrices $T, U, P$ and $Q$ for the linear regression as Equations (4)

$$
Y=X B+B_{o}
$$

where $B$ and $B_{o}$ is PLS regression coefficient. In this research, the coefficients of PLS regression were generated by using the MATLAB matrix routines function. Latent variable (LVs) can be extracted from PLS algorithm after the model was complete. The NIPALS algorithm is the default algorithm applied in PLS [16]. The LVs from NIPALS algorithm were further fed to ANN as the input to predict blood hemoglobin concentrations.

\subsection{Artificial Neural Network}

ANN as one of the nonlinear methods proved their superiority over linear method for making a calibration model [17]. In this research, multi-layer perceptron (MLP) trained with backpropagation algorithm was used as the ANN configuration $[18,19]$. MLP has an input layer of source nodes, hidden layer of neurons, and an output layer of outcome network. Each neuron in hidden layer will receive input parameters of latent variables (LVs) from PLS model. Here, we have measured 1 to 50 number of LVs from PLS model and 1 to 10 number of hidden neurons in order to obtain optimal performance of predictions. Outputs of each $i$ th neuron $x_{i}$ in hidden layer were established by multiplying the corresponding weights with input parameters before passed through a transfer function to get the desired output. The process of transfering information between the input layer and hidden layers is as in Equation (5).

$$
x_{k}=f_{1}\left(\sum_{j=1}^{n} W_{1, k, j}^{i} L V s_{j}+b_{1}\right)
$$

where $f_{1}$ is the hidden layer transfer function, $W_{1, k, j}^{i}$ is the weight of the $k$ th node in the hidden layer connected with the $j$ th node in the input layer. While $b_{1}$ is the bias between hidden layer and input layer. In this study, we use tan-sigmoid transfer functions for hidden layer and linear transfer function for output layer to receive sums of weighed and bias input. To optimize the random initial weights, the network was trained 1000 times to achieve global prediction performance [20]. Levenberg Marquardt (LM) backpropagation algorithm was selected as training algorithm in this study [21]. The training process will stop when either the maximum number of epochs is reached, the goal performance is achieved, the performance of gradient is below minimum gradient value, the momentum update is exceeded, or the failure validation is more than the maximum amount.

\subsection{Performance Validation}

Training and testing performance of prediction were evaluated using root mean squared error of calibration (RMSEC) and root mean squared error of prediction (RMSEP), respectively. RMSEC and RMSEP are computed using Equation (6).

$$
\text { RMSECV or RMSEP }=\sqrt{\frac{\sum_{i=1}^{n}\left(\hat{y}_{i}-y_{i}\right)^{2}}{n}}
$$


where $n$ is the total number of samples, while $\hat{y}_{i}$ and $y_{i}$ denote the predicted blood hemoglobin and reference blood hemoglobin, respectively. To interpret proportion of the variance in the predicted data from the reference value and to describe the relationship between blood hemoglobin and near-infrared spectrum, the coefficient of determination $\left(R^{2}\right)$ was used as Equation (7).

$$
R_{c}^{2} \text { or } R_{p}^{2}=1-\frac{\sum\left(y_{i}-\hat{y}_{i}\right)^{2}}{\sum\left(y_{i}-\bar{y}\right)^{2}}
$$

where $\bar{y}$ is mean of reference data, $\hat{y}_{i}$ and $y_{i}$ denote the unseen predicted and reference blood $\mathrm{Hb}$, respectively.

\section{RESULTS AND DISCUSSION}

\subsection{Latent Variable}

Figure 1 shows the value of RMSEP of PLS and PLS-ANN when a different number of latent variables (LVs) were applied with a different type of SG preprocessing. PLS-ANN without SG preprocessing tends to reach minimum $0.4368 \mathrm{gd} / \mathrm{L}$ of RMSEP when 23 number of the LVs was used. After that, the network becomes overfit when increasing more than 23 number of LVs. Meanwhile, PLS without SG preprocessing shows the further low performance prediction with $0.5307 \mathrm{gd} / \mathrm{L}$ of RMSEP when 14 number of LVs were used. However, there are not many different values of RMSEP for PLS and PLS-ANN when the number of LVs used is between 11 and 16. The effects of SG preprocessing can be seen when smoothing SG preprocessing coupled with PLS-ANN has improved its prediction performance with $0.4208 \mathrm{gd} / \mathrm{L}$ of RMSEP when 26 number of LVs used. In addition, smoothing SG coupled with PLS shows improvement with 0.5114 $\mathrm{gd} / \mathrm{L}$ of RMSEP when 17 number of LVs used. However, the model became overfit when more than 17 number of LVs were used. Meanwhile, first order SG derivatives coupled with PLS-ANN shows an improvement of prediction when achieves $0.4089 \mathrm{gd} / \mathrm{L}$ of RMSEP when 27 number of LVs were used. An improvement also occurs when first order SG derivatives coupled with PLS tends to achieve $0.5024 \mathrm{gd} / \mathrm{L} \mathrm{of}$ RMSEP when 11 number of LVs were used. PLS coupled with second order SG derivative shows increasing of prediction when $0.4669 \mathrm{gd} / \mathrm{L}$ of RMSEP when 14 number of LVs were used. However, PLS-ANN coupled with second order SG derivative shows decreasing of prediction when $0.4295 \mathrm{gd} / \mathrm{L}$ of RMSEP when 19 number of LVs were used.

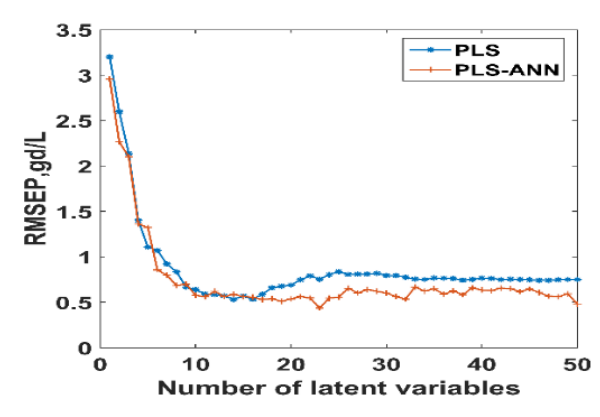

(a)

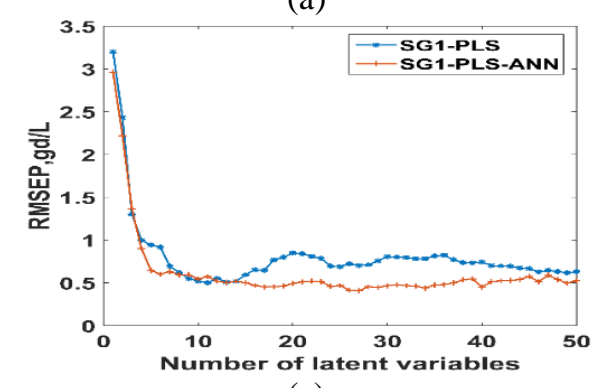

(c)

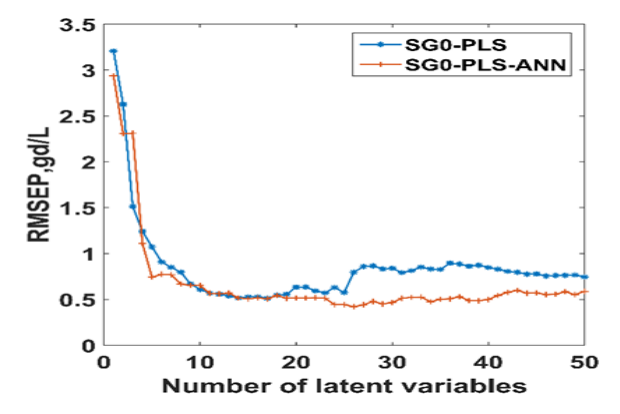

(b)

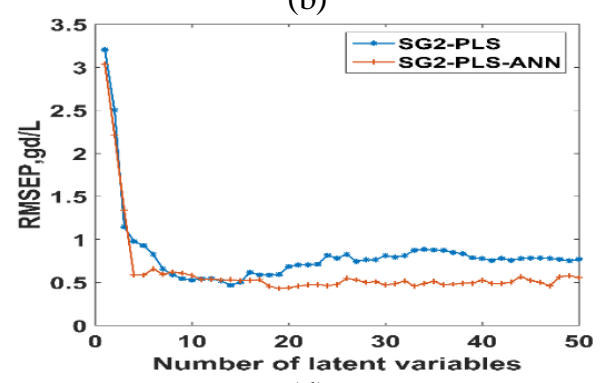

(d)

Figure 1. The root mean square error of prediction of artificial neural network (ANN) and partial least square - artificial neural network (PLS-ANN) versus the change of a number of hidden neurons with different Savitzky-golay (SG) preprocessing: (a) without SG preprocessing, (b) smoothing SG, (c) first order SG derivative, and (d) second order SG derivative 
From the results, we can summarize that PLS coupled with or without SG preprocessing tends to achieve optimal prediction of performance when 11 to 17 number of LVs_were used. While PLS-ANN coupled with or without SG preprocessing tends to achieve optimal prediction of performance with a high number of a LVs when 19 to 26 number of LVs were used. This indicated that the number of LVs used in PLS-ANN need to be optimized to achieve optimal prediction of performance. These results also indicated that PLS-ANN outperforms PLS performance of prediction.

We also observed that with increasing number of LVs, PLS-ANN tends to maintain the value of RMSEP compared to PLS. For instance, PLS-ANN without preprocessing overfitted when 23 number of LVs were used. However, the overfit percentage of PLS-ANN without preprocessing (increasing $49.9 \%$ from 0.4368 to $0.6547 \mathrm{gd} / \mathrm{L}$ of RMSEP) is lowest compared to PLS without preprocessing (increasing $57.7 \%$ from 0.5307 to $0.8369 \mathrm{gd} / \mathrm{L}$ of RMSEP). In addition, the overfit percentage of PLS-ANN with smoothing SG (increasing $21.7 \%$ from 0.4208 to $0.5122 \mathrm{gd} / \mathrm{L}$ of RMSEP) is lowest compared to PLS with first order SG (increasing $67.9 \%$ from 0.5114 to $0.8588 \mathrm{gd} / \mathrm{L}$ of RMSEP). Furthermore, the overfit percentage of PLS-ANN with first order SG derivatives (increasing 33.9\% from 0.4089 to $0.5477 \mathrm{gd} / \mathrm{L}$ of RMSEP) is lowest compared to PLS with first order SG derivatives (increasing $69.2 \%$ from 0.5024 to $0.85 \mathrm{gd} / \mathrm{L}$ of RMSEP). Then, the overfit percentage of PLS-ANN with second order SG derivatives (increasing $27.4 \%$ from 0.4295 to $0.5473 \mathrm{gd} / \mathrm{L}$ of RMSEP) is lowest compared to PLS with second order SG derivatives (increasing $77 \%$ from 0.4669 to $0.8263 \mathrm{gd} / \mathrm{L}$ of RMSEP). These findings indicated that PLS-ANN has the minimum potential to be overfitted compared to PLS. Moreover, PLS-ANN managed to obtain a better result compared to PLS. This shows that feedforward backpropagation of ANN has had a good impact on improving prediction on PLS model. These findings agree with previous research that PLS-ANN results in a better performance than that of PLS [10, 21].

\subsection{Hidden Neurons}

Figure 2 shows the RMSEP of PLS and PLS-ANN when different number of hidden neurons was applied with different type of SG preprocessing. PLS-ANN without SG preprocessing tends to achieve optimum performance of prediction with $0.4368 \mathrm{gd} / \mathrm{L}$ of RMSEP when 5 number of hidden neurons were used. After that, the network is overfitting when more than 5 number of hidden neurons were used. Meanwhile, ANN without SG preprocessing is able to achieve optimum performance of prediction with $0.4607 \mathrm{gd} / \mathrm{L}$ of RMSEP when 3 number of hidden neurons were used. Smoothing SG coupled with ANN and smoothing SG coupled with PLS-ANN tends to achieve optimal prediction performance when 2 number of hidden were used with $0.4208 \mathrm{gd} / \mathrm{L}$ and $0.4499 \mathrm{gd} / \mathrm{L}$ of RMSEP respectively. However, the network is overfitted when more than 2 number of hidden neurons were used. First order SG derivatives coupled with ANN show a better result with optimum performance of prediction with $0.4089 \mathrm{gd} / \mathrm{L}$ of RMSEP when 5 number of hidden neurons was used compared with first order SG derivatives coupled with PLS-ANN.

Second order SG derivatives coupled with PLS-ANN tend to achieve optimal prediction performance $0.4295 \mathrm{gd} / \mathrm{L}$ of RMSEP when 2 number of hidden neurons were used. After that, the network is overfitted when more than 2 number of hidden neurons were used. Meanwhile, second order SG derivatives coupled with ANN achieve the lower performance of prediction with $0.4571 \mathrm{gd} / \mathrm{L}$ of RMSEP when 3 hidden neurons were used compared to second order SG derivatives coupled with PLS-ANN.

Despite with different SG preprocessing, PLS-ANN could obtain a better performance of prediction compared to ANN except first order SG derivatives preprocessing. However, first order SG derivatives coupled with ANN can be considered as the best model with the high performance of prediction $0.4089 \mathrm{gd} / \mathrm{L}$ of RMSEP when 5 number of hidden neurons were used. This finding indicates that the number of LVs was used as input of ANN is not enough to represent important information the whole spectral data itself. Therefore, ANN with directly receiving input from spectral data after treated tends to achieve a better performance of prediction compared to PLS-ANN with LVs as the inputs of ANN. 


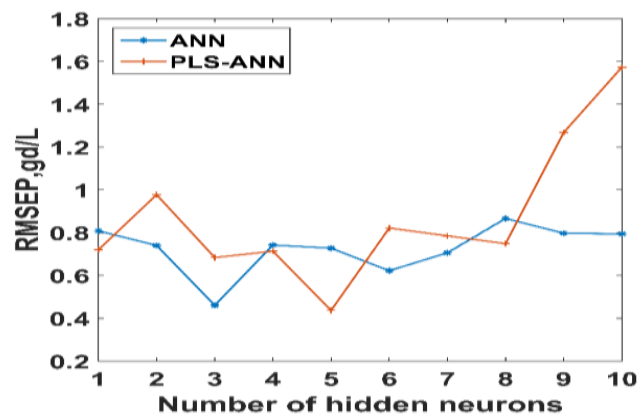

(a)

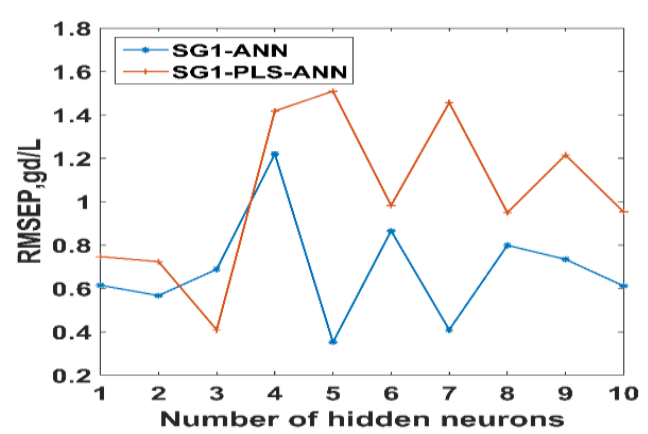

(c)

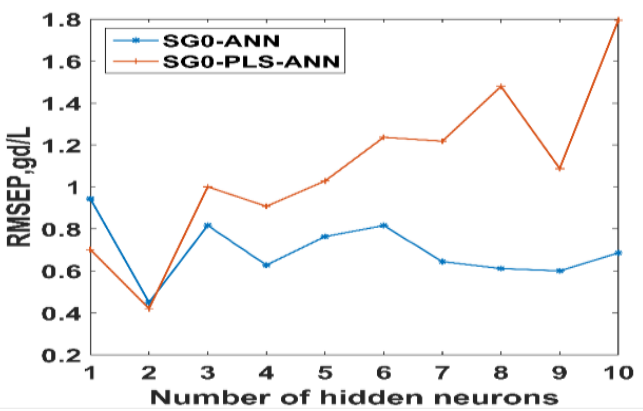

(b)

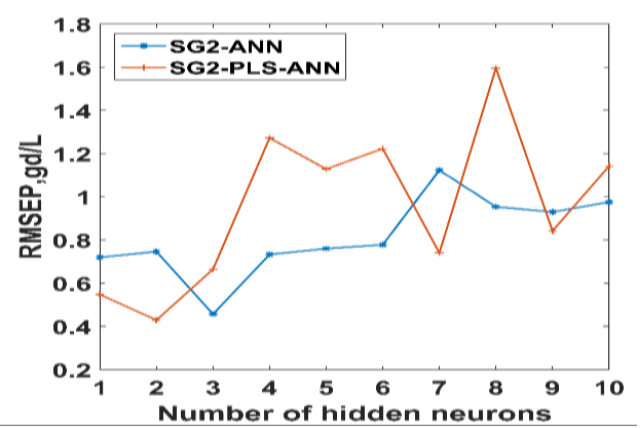

(d)

Figure 2. The root mean square error of prediction of artificial neural network (ANN) and partial least square - artificial neural network (PLS-ANN) versus the change of a number of hidden neurons with different Savitzky-golay (SG) preprocessing: (a) without SG preprocessing, (b) smoothing SG, (c) first order SG derivative, and (d) second order SG derivative

\subsection{Prediction Performance}

Table 2 shows the accuracy of the PLS, ANN, and PLS-ANN with different SG preprocessing methods using fixed number of frame length that is smoothing $=77 \mathrm{~nm}$, first order SG derivatives $=27 \mathrm{~nm}$, and second order SG derivatives $=79 \mathrm{~nm}$ with the optimal number latent variables (LVs). Best prediction of performance was achieved by ANN coupled that with first order SG derivatives with RMSEP of $0.3517 \mathrm{gd} / \mathrm{L}$ and $R_{p}^{2}$ of 0.9849 , followed by PLS-ANN that coupled with first order SG derivative with RMSEP of 0.4089 $\mathrm{gd} / \mathrm{L}$ and $R_{p}^{2}$ of 0.9792 , and then PLS that coupled second order SG derivative with RMSEP of $0.4669 \mathrm{gd} / \mathrm{L}$ and $R_{p}^{2}$ of 0.9727 . This unusual result whereas conventional model tends to give a better result compared hybrid model is a normal finding in multivariate calibration analysis. For instance, auto regressive integrated moving average and ANN (ARIMA_ANN) are marginally showed better performance then hybrid ANN_ARIMA in Indian stock trend forecasting research [22]. Moreover, ANN showed a slightly higher performance in term of $R^{2}$ and lower RMSE compared to PLS-ANN in consumer liking scores of ready-todrink green tea beverages predictions [9]. Furthermore, the criteria of datasets with extrapolation samples may influenced the prediction performance of different predictive modelling. However, PLS-ANN that coupled with smoothing, first order, second order SG derivative and without SG preprocessing showed satisfied result by dominating top best five of RMSEP that were $0.4208,0.4089,0.4295$, and $0.4368 \mathrm{gd} / \mathrm{L}$, respectively. Although ANN outperformed PLS-ANN, PLS-ANN is simpler than ANN with three hidden neurons compared to the former that needed five hidden neurons to achieve the optimal performance.

The result also indicated that the PLS model was found to have the lowest prediction performance while ANN and PLS-ANN hybrid models were found to have comparable qualities based on the values of $R_{p}^{2}$ and RMSEP. This result suggested that the linear PLS model cannot generalize nonlinearity of spectral data and these findings are in line with the results of the previous study [9]. While the characteristics of ANN modelling that can adapt and generalize data sets having non-linear relationships makes ANN achieve a better result in this research. In addition, PLS that coupled with second order SG derivative achieved the optimal accuracy compared to that coupled with smoothing and first order SG derivatives is in line with previous study [14]. Moreover, ANN that coupled with the first order SG derivative with a proper optimization was able to achieve a better predictive accuracy in predicting the blood hemoglobin using nearinfrared spectral data with RMSEP of $0.3517 \mathrm{gd} / \mathrm{L}$ and of 0.9849 , compared with the previous works [13]. 
Table 2. The accuracy of the PLS, ANN, and PLS-ANN with different SG preprocessing methods using the optimal tunable parameters of frame length, LVs, and hidden neurons

\begin{tabular}{|c|c|c|c|c|c|c|c|c|}
\hline $\begin{array}{l}\text { Modelling } \\
\text { Method }\end{array}$ & SG preprocessing method & $\begin{array}{l}\text { Frame } \\
\text { length }\end{array}$ & $\begin{array}{c}\text { Latent } \\
\text { variable }\end{array}$ & $\begin{array}{l}\text { Hidden } \\
\text { neurons }\end{array}$ & \multicolumn{2}{|c|}{ Training } & \multicolumn{2}{|c|}{ Prediction } \\
\hline \multirow{3}{*}{ PLS } & Without preprocessing & N/A & 14 & N/A & 0.3354 & 0.9588 & 0.5307 & 0.9648 \\
\hline & First order SG derivative & 27 & 11 & N/A & 0.3335 & 0.9592 & 0.5024 & 0.9684 \\
\hline & Second order SG derivative & 79 & 14 & N/A & 0.3287 & 0.9604 & 0.4669 & 0.9727 \\
\hline \multirow{2}{*}{ ANN } & Without preprocessing & N/A & N/A & 3 & 0.1830 & 0.9890 & 0.4607 & 0.9734 \\
\hline & Second order SG derivative & 79 & N/A & 3 & 0.1190 & 0.9950 & 0.4571 & 0.9746 \\
\hline \multirow{4}{*}{ PLS-ANN } & Without preprocessing & N/A & 23 & 5 & 0.1364 & 0.9933 & 0.4368 & 0.9787 \\
\hline & SG smoothing & 77 & 26 & 2 & 0.1586 & 0.9908 & 0.4208 & 0.9784 \\
\hline & First order SG derivative & 27 & 27 & 3 & 0.1473 & 0.9923 & 0.4089 & 0.9792 \\
\hline & Second order SG derivative & 79 & 19 & 2 & 0.2554 & 0.9760 & 0.4295 & 0.9782 \\
\hline
\end{tabular}

\section{CONCLUSION}

This study shows that the hybrid model (i.e. PLS-ANN) that combined linear model (i.e. PLS) and nonlinear model (i.e. ANN) achieved satisfying results in predicting the blood Hemoglobin using nearinfrared spectral data. ANN that coupled with first order SG derivatives achieved the best prediction with RMSEP of $0.3517 \mathrm{gd} / \mathrm{L}$ and $R_{p}^{2}$ of 0.9849 , followed by PLS-ANN that coupled with first order SG derivative with RMSEP of $0.4368 \mathrm{gd} / \mathrm{L}$ and $R_{p}^{2}$ of 0.9787 , and PLS coupled second order SG derivative with RMSEP of $0.4669 \mathrm{gd} / \mathrm{L}$ and $R_{p}^{2}$ of 0.9727 . This suggests that the related spectrum information may be excluded in the first few latent variables of PLS. Nevertheless, findings indicated that PLS-ANN was able to minimize the overfitting problem compared to PLS with fewer input variables. Furthermore, the structure of PLS-ANN is simpler than ANN, in which, the former needed three hidden neurons compared with the latter needed five hidden neurons in achieving their optimal prediction performance. Thus, a nonlinear model is crucial to model these nonlinear information in NIR spectroscopic analysis and more researches are required in understanding the potential of hybrid models in in NIR spectroscopic analysis.

\section{ACKNOWLEDGMENTS}

The author would like to acknowledge the Ministry of Education Malaysia and Research Management Centre (RMC), Universiti Tun Hussein Onn Malaysia (UTHM) for financial support GPP H312 and providing facilities for this study.

\section{REFERENCES}

[1] V. Gomes, et al., "Characterization of neural network generalization in the determination of $\mathrm{pH}$ and anthocyanin content of wine grape in new vintages and varieties," Food Chemistry, vol. 218, pp. 40-46, 2017.

[2] Y. Zhang, et al., "Comparision of Several Preprocessing Algorithms Based on Near Infrared Spectroscopic Measurement of Glucose in Aqueous Glucose Solutions," TELKOMNIKA Telecommunication Computing Electronics and Control, vol. 12, pp. 2683-2689, 2014.

[3] H. A. G. Al-kaf, et al., "A comparison between single layer and multilayer artificial neural networks in predicting diesel fuel properties using near infrared spectrum," Petroleum Science and Technology, vol. 6466, pp. 1-8, 2018.

[4] M. Pojić, et al., "The Application of Near Infrared Spectroscopy in Wheat Quality Control," Infrared Spectroscopy - Life and Biomedical Sciences, vol. 1, pp. 167-184, 2012.

[5] J. Yadav, et al., "Prospects and limitations of non-invasive blood glucose monitoring using near-infrared spectroscopy," Biomedical Signal Processing and Control, vol. 18, pp. 214-227, 2015.

[6] K. D. Chito, et al., "Incremental model learning for spectroscopy-based food analysis," Chemometrics and Intelligent Laboratory Systems, vol. 167, pp. 123-131, 2017.

[7] M. Khashei, et al., "A novel hybrid classification model of artificial neural networks and multiple linear regression models," Expert Systems with Applications, vol. 39, pp. 2606-2620, 2012.

[8] K. Song, et al., "Remote sensing of chlorophyll-a concentration for drinking water source using genetic algorithms (GA)-partial least square (PLS) modeling," Ecological Informatics, vol. 10, pp. 25-36, 2012.

[9] P. Yu, et al., "Development of a partial least squares-artificial neural network (PLS-ANN) hybrid model for the prediction of consumer liking scores of ready-to-drink green tea beverages," Food Research International, vol. 103, pp. 68-75, 2018.

[10] H. Huang, "A Hybrid Neural Network Prediction Model of Air Ticket," TELKOMNIKA Telecommunication Computing Electronics and Control, vol. 11, pp. 6413-6419, 2013. 
[11] S. Li, et al., "Hybridization of partial least squares and neural network models for quantifying lunar surface minerals," Icarus, vol. 221, pp. 208-225, 2012.

[12] S. Hosseinpour, et al., "Exact estimation of biodiesel cetane number (CN) from its fatty acid methyl esters (FAMEs) profile using partial least square (PLS) adapted by artificial neural network (ANN)," Energy Conversion and Management, vol. 24, pp. 389-398, 2016.

[13] B. Igne, et al., "The 2010 IDRC software shoot-out at a glance," NIR news, vol. 21, pp. 14, 2010.

[14] K. S. Chia, et al., "Evaluation of common pre-processing approaches for visible (VIS) and shortwave near infrared (SWNIR) spectroscopy in soluble solids content (SSC) assessment," Biosystems Engineering, vol. 115, pp. 82-88, 2013.

[15] J. Luo, et al., "Properties of Savitzky-Golay digital differentiators," Digital Signal Processing: A Review Journal, vol. 15, pp. 122-136, 2005.

[16] S. Wold, et al., "PLS-regression: A basic tool of chemometrics," Chemometrics and Intelligent Laboratory Systems, vol. 58, pp. 109-130, 2001.

[17] R. M. Balabin, et al., "Comparison of linear and nonlinear calibration models based on near infrared (NIR) spectroscopy data for gasoline properties prediction," Chemometrics and Intelligent Laboratory Systems, vol. 88, pp. 183-188, 2007.

[18] D. Svozil, et al., "Introduction to multi-layer feed-forward neural networks," Chemometrics and Intelligent Laboratory Systems, vol. 39, pp. 43-62, 1997.

[19] S. Haykin, "Feedforward Neural Networks: An Introduction," Convergence, pp. 1-16, 1998.

[20] G. Binetti, et al., "Cultivar classification of Apulian olive oils: Use of artificial neural networks for comparing NMR, NIR and merceological data," Food Chemistry, vol. 219, pp. 131-138, 2017.

[21] H. Karim, et al., "Comparison of Neural Network Training Algorithms for Classification of Heart Diseases," IAES International Journal of Artificial Intelligence, vol. 7, pp. 185-189, 2018.

[22] N. Merh, et al., "A Comparison Between Hybrid Approaches of Ann and Arima for Indian Stock Trend Forecasting," Business Intelligence Journal, pp. 23-43, 2010. 